Article

\title{
Bacterial Production, Characterization and Protein Modeling of a Novel Monofuctional Isoform of FAD Synthase in Humans: An Emergency Protein?
}

\author{
Piero Leone ${ }^{1,+}{ }^{,}$Michele Galluccio ${ }^{2,+}{ }^{(\mathbb{D}}$, Alberto Barbiroli ${ }^{3}$, Ivano Eberini ${ }^{4}$ (D), Maria Tolomeo ${ }^{1}$, \\ Flavia Vrenna ${ }^{2}$, Elisabetta Gianazza ${ }^{4}$, Stefania Iametti ${ }^{3}$ (D), Francesco Bonomi ${ }^{3}$, \\ Cesare Indiveri $^{2,5}$ and Maria Barile ${ }^{1, *}$ \\ 1 Department of Bioscience, Biotechnology and Biopharmaceutics, University of Bari, via Orabona, 4, \\ I-70126 Bari, Italy; pieroleone87@gmail.com (P.L.); maria.tolomeo89@gmail.com (M.T.) \\ 2 Department of Biology, Ecology and Earth Science (DiBEST), Unit of Biochemistry and Molecular \\ Biotechnology, University of Calabria, Via P. Bucci 4c, I-87036 Arcavacata di Rende, Italy; \\ michele.galluccio@unical.it (M.G.); flaviavrenna@libero.it (F.V.); cesare.indiveri@unical.it (C.I.) \\ 3 Dipartimento di Scienze per gli Alimenti, la Nutrizione e l'Ambiente (DeFENS), Università degli Studi di \\ Milano, via G. Celoria 2, I-20133 Milano, Italy; alberto.barbiroli@unimi.it (A.B.); \\ stefania.iametti@unimi.it (S.I.); francesco.bonomi@unimi.it (F.B.) \\ 4 Gruppo di Studio per la Proteomica e la Struttura di Proteine, Dipartimento di Scienze Farmacologiche e \\ Biomolecolari (DiSFeB), Università degli Studi di Milano, via Balzaretti 9, I-20133 Milano, Italy; \\ ivano.eberini@icloud.com (I.E.); elisabetta.gianazza@unimi.it (E.G.) \\ 5 Institute of Biomembranes, Bioenergetics and Molecular Biotechnology (IBIOM) - CNR, Via Giovanni \\ Amendola 165/A-70126 Bari, Italy \\ * Correspondence: maria.barile@uniba.it; Tel.: +39-080-5443-604 \\ + These authors contributed equally to this work.
}

Received: 2 December 2017; Accepted: 4 January 2018; Published: 6 January 2018

\begin{abstract}
FAD synthase (FADS, EC 2.7.7.2) is the last essential enzyme involved in the pathway of biosynthesis of Flavin cofactors starting from Riboflavin (Rf). Alternative splicing of the human FLAD1 gene generates different isoforms of the enzyme FAD synthase. Besides the well characterized isoform 1 and 2, other FADS isoforms with different catalytic domains have been detected, which are splice variants. We report the characterization of one of these novel isoforms, a 320 amino acid protein, consisting of the sole C-terminal $3^{\prime}$-phosphoadenosine $5^{\prime}$-phosphosulfate (PAPS) reductase domain (named FADS6). This isoform has been previously detected in Riboflavin-Responsive (RR-MADD) and Non-responsive Multiple Acyl-CoA Dehydrogenase Deficiency (MADD) patients with frameshift mutations of FLAD1 gene. To functionally characterize the hFADS6, it has been over-expressed in Escherichia coli and purified with a yield of $25 \mathrm{mg} \cdot \mathrm{L}^{-1}$ of cell culture. The protein has a monomeric form, it binds FAD and is able to catalyze FAD synthesis $\left(k_{\text {cat }}\right.$ about $\left.2.8 \mathrm{~min}^{-1}\right)$, as well as FAD pyrophosphorolysis in a strictly $\mathrm{Mg}^{2+}$-dependent manner. The synthesis of FAD is inhibited by $\mathrm{HgCl}_{2}$. The enzyme lacks the ability to hydrolyze FAD. It behaves similarly to PAPS. Combining threading and ab-initio strategy a 3D structural model for such isoform has been built. The relevance to human physio-pathology of this FADS isoform is discussed.
\end{abstract}

Keywords: flavin; riboflavin; flavoprotein; FAD synthase; protein modeling; over-expression; E. coli

\section{Introduction}

Riboflavin, or vitamin B2, is the precursor of flavin mononucleotide (FMN) and flavin adenine dinucleotide (FAD), which are essential cofactors of hundreds of oxidoreductases (EC1) but also of other flavoproteins and flavoenzymes which are not oxidoreductases. These enzymes are involved in 
cellular metabolism and in several regulatory pathways, such as antioxidant defense, protein folding, chromatin remodeling and apoptosis [1-4].

These flavoenzymes are mainly located in the cellular organelles [5], where they ensure the functionality of the mitochondrial respiratory chain and of the Krebs cycle, and the metabolism of fatty acids, of some amino acids, of choline and betaine [6-8], and the synthesis of protoporphyrin [9]. Flavoenzymes are also implicated in the tetrahydrofolate-dependent one-carbon metabolism, as well as in the metabolism of vitamins B6, B9, and B12 [10-13].

Whereas bacteria are able to synthesize and export riboflavin $[14,15]$, humans must obtain it as an essential nutrient via intestinal absorption from food or from the intestinal microbiota $[16,17]$. Riboflavin is transported into the bloodstream and taken up by target cells by the three human riboflavin transporters (RFVTs) recently identified as members of the solute carrier family SLC52A [10]. Mutations in RFVTs are causative of a rare neurodegenerative disorder named Brown-Vialetto-Van Laere syndrome and of a transient infantile form of RR-MADD [18-20].

Once in the cell, riboflavin is converted to FMN by riboflavin kinase (RFK, EC2.7.1.26) and FMN is converted, in turn, to FAD by a distinct enzyme, namely FMN:ATP adenylyl transferase, commonly known as FAD synthase (FADS, EC 2.7.7.2) or FAD synthetase [21,22]. Differently from mammals, prokaryotes synthesize FAD using a single bi-functional enzyme, which presents a C-terminal riboflavin kinase activity and a N-terminal FMN-adenylyl transferase (FMNAT) activity [23,24].

In humans, distinct isoforms of FADS are the products of the FLAD1 gene (MIM: 610595), which is alternatively spliced in different transcript variants (Figure 1) that can be visualized by the UCSC (University of California Santa Cruz) Genome Browser. Two FADS isoforms deriving from the translation of seven exon long mRNAs have been characterized in detail $[22,25,26]$. The longest protein, namely FADS1, has a predicted mitochondrial targeting sequence (MTS), and a mitochondrial localization [27]. A slightly shorter isoform derives from interruption of exon 1 by an additional intron. This leads to usage of a downstream ATG coding Met98 of the longer transcript, resulting in FADS2 (Figure 1), with cytosolic localization; this 490 residues long enzyme was proposed to be a component of the FAD delivery machinery [25].

Both FADS isoforms contain a N-terminal molybdopterin binding (MPTb) domain, which has been shown to have FAD hydrolase activity [28], and a C-terminal 3-phosphoadenosine 5-phosphosulfate (PAPS) reductase domain, which per se performs the FAD synthase activity [29].

The expected central role of FADS in human muscle bioenergetics has been recently proved by the occurrence of certain riboflavin-responsive and non-responsive cases of Multiple Acyl CoA dehydrogenase deficiency (MADD, OMIM 231680) and/or of multiple respiratory-chain deficiency caused by mutations in FLAD1 [30,31]. The affected individuals show a severe metabolic myopathy, resembling what is seen in individuals with Electron Transfer Flavoprotein Dehydrogenase (ETFDH) defects [31-33]. Interestingly, the residual FADS activity detectable in these patients with homozygous frameshift variants in the MPTb domain led to the discovery of novel transcript isoforms, whose existence was validated by transcriptomic analysis. Theoretically, in humans, novel FADS isoforms, starting at Met268 (Figure 1) and at Met355, with respect to isoform 1, could be translated from the novel variant FLAD1 transcripts.

Since FLAD1 gene is the sole gene coding for FADS, these novel mono-functional isoforms, lacking of the MPTb domain, i.e., containing only the PAPS domain, might ensure cytosolic FAD synthesis and explain why affected individuals with biallelic FLAD1 frameshift variants still harbor substantial FADS activity and thus survive.

Even if the existence of these protein products was detected in MADD patient fibroblasts [30], the actual existence, the identity, and the functional and structural features of these isoforms are still a matter of investigation. 
In the investigation accounted for in this report, we have over-produced in E. coli and purified with a yield sufficient to allow functional characterization and structure/function relationships studies the novel putative natural isoform starting at Met268 and named hFADS6. This isoform is able to perform both $\mathrm{Mg}^{2+}$-dependent FAD synthesis and the reverse reaction (i.e., FAD pyrophosphorolysis), but not FAD hydrolysis, the latter finding correlating with the lack of the molybdopterin binding domain in the novel variant (Figure 1).

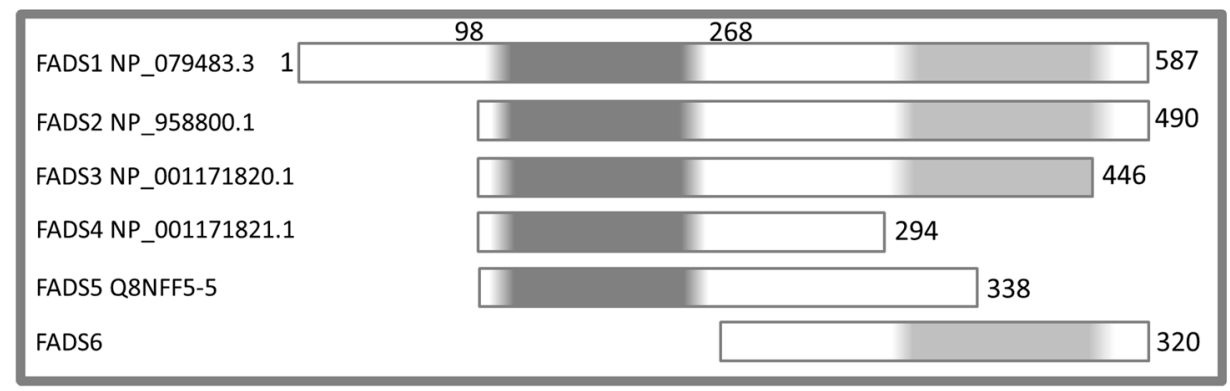

Figure 1. Schematic representation of the human FAD synthase protein isoforms. The protein length and the enzymatic activities of six human FAD synthase isoforms are reported. Molybdopterin binding-like domain, responsible of FAD hydrolysis, is indicated in dark grey (N-terminus); phosphoadenylyl sulfate reductase domain (PAPS), competent in FAD synthesis and in pyrophosphorolysis, is highlighted in light grey (C-terminus). hFADS1, hFADS2 and hFADS3 possess both catalytic domains; hFADS4 and hFADS5 present the sole molybdopterin binding domain; on the contrary, hFADS6 has the sole PAPS reductase domain. Numbers indicate amino acid positions in the resulting protein sequence of the FADS1 isoform.

\section{Results}

\subsection{Cloning, Expression and Purification of the hFADS6 Isoform}

The cDNA coding for human FADS isoform 6 (hFADS-6) was cloned between EcoRI and XhoI restriction sites of the pH6EX3 expression vector, generating a fusion peptide with a N-terminal 6-His-tag, whose theoretical molecular mass is $38.266 \mathrm{kDa}$. To optimize the heterologous production of the protein, several E. coli strains were screened for transformation, i.e., BL21(DE3)pLysS, JM109, RosettaGami2(DE3)pLysS, Rosetta(DE3)pLysS. E. coli Rosetta(DE3)pLysS strain, that is supplemented with transfer RNA (tRNA)-specific for rare codons, emerged as the best choice in terms of production of the recombinant protein (Figure 2).

Indeed, a band at about $36.0 \mathrm{kDa}$ was present in the induced cell lysate after $2 \mathrm{~h}$ induction (Figure 2A lane 2), which was absent in non-induced cell lysate and supernatant (Figure 2A lanes 1 and 3, respectively). Different growth conditions and Isopropyl $\beta$-D-1-thiogalactopyranoside (IPTG) concentrations were tested to improve expression; IPTG concentration from $0.1 \mathrm{mM}$ to $1 \mathrm{mM}$ did not influence protein expression (not shown). The cell lysates were separated in a soluble and insoluble cell fraction as described in Materials and Methods. The amount of expressed protein recovered in the soluble fraction increased with time of growth (Figure 2A lanes 4-6). However, after $4 \mathrm{~h}$, which was the best condition (Figure 2A, lane 5), protein degradation was also observed (Figure 2A lane 6), which was not prevented by lowering the temperature to $20^{\circ} \mathrm{C}$ (Figure $2 \mathrm{~A}$, lane 7 ). A lower amount of protein was recovered in the cell pellet after $4 \mathrm{~h}$ induction (Figure 2A, lane 8), accounting for about $30 \%$ of the protein in the soluble fraction. The protein was identified by immunoblot performed with both the in-house produced anti-FADSs antibody (Figure 2B lane $1^{\prime}$ and $5^{\prime}$ ) and the anti-His antibody as a control (Figure 2C, lanes $1^{\prime}$ and $5^{\prime}$ ). In both cases a strong immunoreaction was observed in the induced cell lysate, which was virtually absent in the non-induced lysates. The faint immunoreaction at higher molecular mass might be due to aggregation of a very small fraction of the protein. 
To purify the protein of interest, the soluble fraction, corresponding to lane 5 of Figure 2 and to lane 1 of Figure 3, was applied on a $\mathrm{Ni}^{2+}$ chelating column and eluted as described in Materials and Methods. The column was then washed with $50 \mathrm{mM}$ imidazole (Figure 3A lane 3); the elution of the 6His-hFADS6 was started with $100 \mathrm{mM}$ imidazole (Figure 3A lanes 4 and 5) and was completed by increasing the imidazole concentration to $250 \mathrm{mM}$. The purified protein resulted in an apparently homogenous band (Figure 3A, lanes 6 and 7). The apparent molecular mass on SDS-PAGE was about $36 \mathrm{kDa}$, very close to the theoretical molecular mass of the 6His-hFADS6. The protein yield was about $25 \mathrm{mg} \cdot \mathrm{L}^{-1}$ of cell culture.
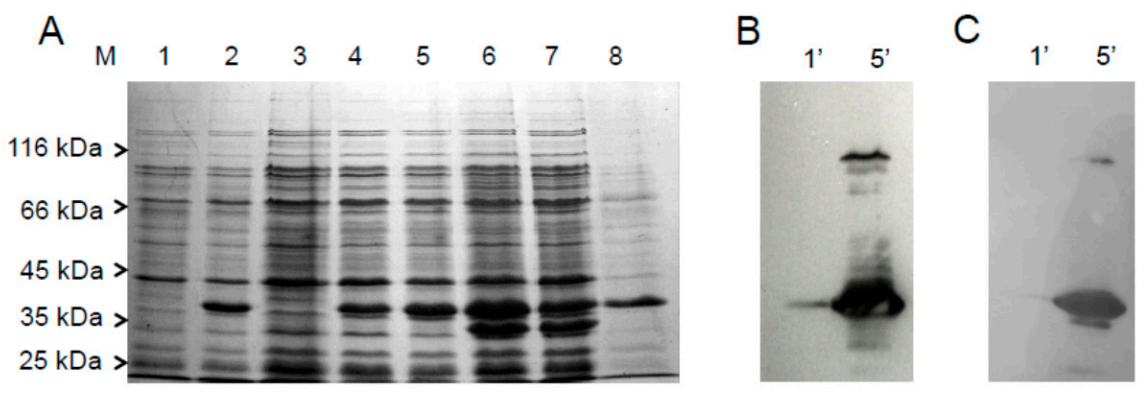

Figure 2. Over-expression and identification of recombinant hFADS6. (A) Proteins were separated by SDS-PAGE (Sodium Dodecylsulfate-Polyacrylamide Gel Electrophoresis) on 12\% T polyacrylamide gel and stained with Coomassie Blue. Lane M: molecular mass markers: beta-galactosidase (116 kDa), bovine serum albumin (BSA) (66 kDa), ovalbumin (45 kDa), lactate dehydrogenase (35 kDa), restriction endonuclease Bsp98I (25 kDa); lane 1, non-induced cell lysate after $2 \mathrm{~h}$ of growth (6 $\mu \mathrm{g})$; lane 2, cell lysate after $2 \mathrm{~h}$ of induction $(8 \mu \mathrm{g})$; lane 3 , soluble fraction of non-induced cell lysate after $2 \mathrm{~h}$ of growth $(10 \mu \mathrm{g})$; lanes $4-6$, soluble fractions of cell lysate after $2 \mathrm{~h}, 4 \mathrm{~h}$ and $8 \mathrm{~h}$ of $0.1 \mathrm{mM}$ isopropyl $\beta$-D-1-thiogalactopyranoside (IPTG) induction at $28^{\circ} \mathrm{C},(12,14$ and $15 \mu \mathrm{g})$ respectively; lane 7 , soluble fractions of cell lysate after $8 \mathrm{~h}$ of $0.1 \mathrm{mM}$ IPTG induction at $20^{\circ} \mathrm{C}(13 \mu \mathrm{g})$; lane 8 , insoluble fraction of cell lysate after $4 \mathrm{~h}$ of $0.1 \mathrm{mM}$ IPTG induction at $28^{\circ} \mathrm{C}(7 \mu \mathrm{g})$; (B) Immunoblotting of hFADS6: lanes $1^{\prime}$ and $5^{\prime}$, immunodetection on the same protein fractions as in (A) with anti-FADS antiserum (1:2500); (C) lanes $1^{\prime}$ and $5^{\prime}$, immunodetection on the same protein fractions as in (A) with anti-His antibody $(1: 40,000)$. The molecular mass markers in $(\mathbf{B}, \mathbf{C})$ were as in $(\mathbf{A})$.

Spectrophotometric analysis of purified fraction 5 (Figure 3B) as well as of fractions 4, 6 and 7 (not shown) showed a typical flavoprotein absorbance spectrum, with a main peak at $274 \mathrm{~nm}$ and two minor peaks at 350 and $450 \mathrm{~nm}$ (Figure 3B) indicating that this novel purified isoform, as the previously characterized isoform 2, tightly binds flavins [25]. High performance liquid chromatography (HPLC) analysis of the supernatant obtained by acidic treatment of purified hFADS6 demonstrates that the bound cofactor is FAD (Figure 3C). The molecular ratio of FAD/monomer as determined by optical properties as well as by HPLC (see Materials and Methods) gave a value of $0.60 \pm 0.015$. 


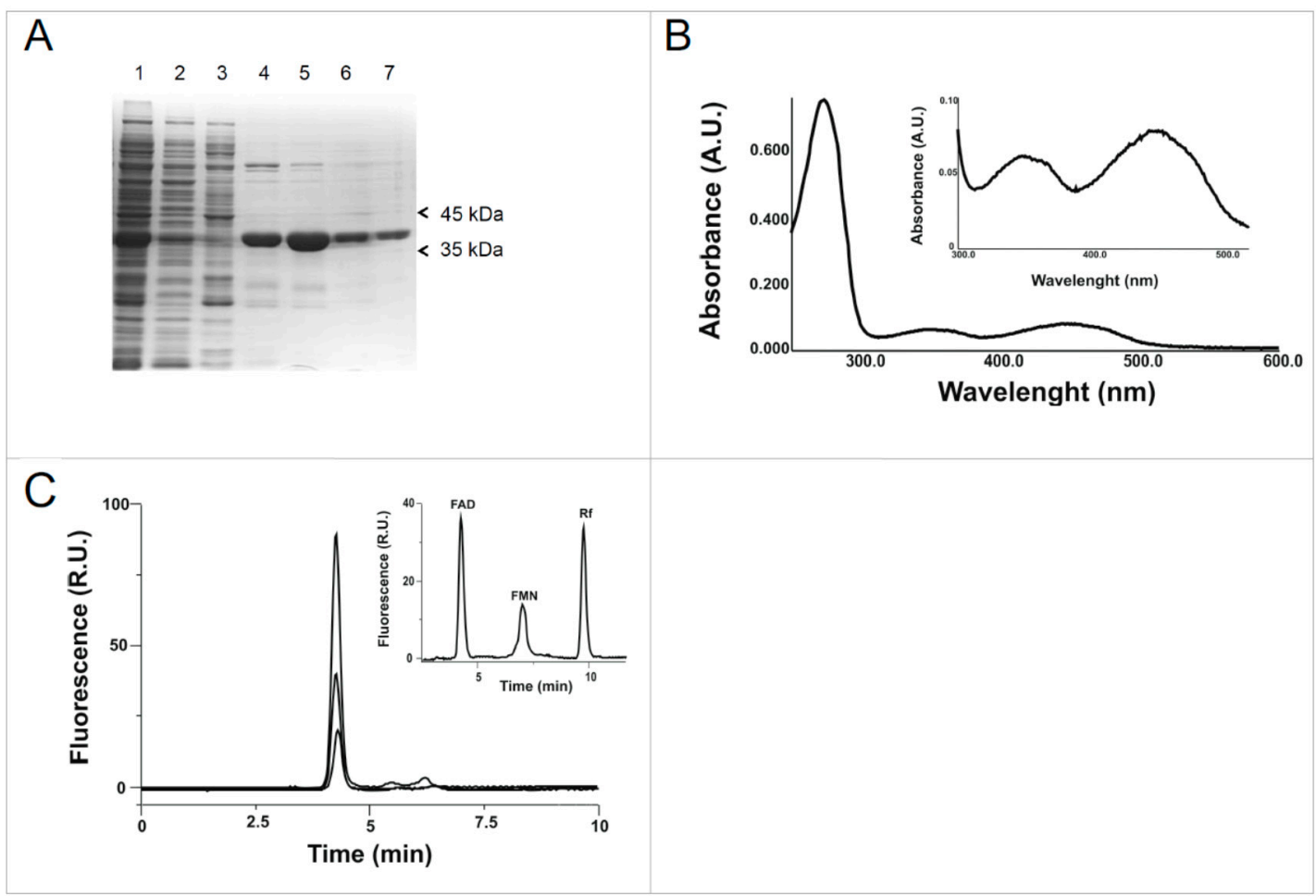

Figure 3. Purification of 6 His-hFADS6. (A) Protein fractions obtained by $\mathrm{Ni}^{2+}$-chelating chromatography were separated by SDS-PAGE on $12 \% \mathrm{~T}$ polyacrylamide gel and stained with Coomassie Blue. Lane 1, soluble fraction of IPTG-induced cell lysate $(30 \mu \mathrm{g})$; lane 2, first flow-through fraction $(10 \mu \mathrm{g})$; lane 3, proteins eluted with $50 \mathrm{mM}$ imidazole $(8 \mu \mathrm{g})$; lane 4 , first fraction of proteins eluted with $100 \mathrm{mM}$ imidazole $(9 \mu \mathrm{g})$; lane 5, second fraction of proteins eluted with $100 \mathrm{mM}$ imidazole $(10 \mu \mathrm{g})$; lane 6 , first fraction of proteins eluted with $250 \mathrm{mM}$ imidazole $(5 \mu \mathrm{g})$; lane 7 , second fraction of proteins eluted with $250 \mathrm{mM}$ imidazole $(4 \mu \mathrm{g})$; (B) Absorbance spectrum of the protein purified to homogeneity (Panel A, lane 7). The spectrum of hFADS6 $(11.5 \mu \mathrm{M})$ was recorded in $40 \mathrm{mM}$ Hepes/Na, $5 \mathrm{mM} \beta$-mercaptoethanol, pH 7.4. In the inset, a zoom in the visible region; (C) HPLC analysis of acid-extractable flavin cofactor. The purified protein $(15.4 \mu \mathrm{g}$ in $100 \mu \mathrm{L}, 4 \mu \mathrm{M})$ was treated with $10 \%$ perchloric acid. The supernatant was neutralized and aliquots (10 and $20 \mu \mathrm{L})$ were analyzed for flavin content by HPLC. In the inset, chromatographic peaks of standard aqueous FAD (40 pmol), FMN (10 pmol) and riboflavin (10 pmol) solutions are shown for comparison.

\subsection{Molecular Characterization of hFADS6}

The aggregation state of hFADS6 and of hFADS2 was assessed by size exclusion chromatography and hydrodynamic radius measurement performed by tandem HPLC-GPC/LS. As shown in Figure 4A, hFADS6 migrated in HPLC-GPC as a single peak with an elution volume of $13 \mathrm{~mL}$, much higher than what observed for hFADS2 (elution volume, $11 \mathrm{~mL}$ ). The addition of $1 \mathrm{mM}$ DTT to the elution buffer did not alter the chromatographic behavior of either protein, if not for improving the sharpness of individual peaks, likely because of the dissociation of minor amounts of disulfide linked aggregates. Online MALS (Multi-Angle Light Scattering) measurements performed on the peaks obtained in the presence of DTT gave molecular masses of $36.5 \pm 5 \mathrm{kDa}$ for hFADS6 and $111 \pm 17 \mathrm{kDa}$ for hFADS2, confirming that the hFADS6 and the hFADS2 had a monomeric and dimeric nature, respectively.

hFADS6 has a CD signal originating from alpha-helical regions in secondary structure, and its CD spectrum nearly overlaps that of hFADS2 (Figure 4B), if not for minor differences in signal intensity, that could be related to the different length of the two isoforms. Within the limitations due to incomplete coverage of the far ultra-violet (UV) region and to the approximations inherent to these models, the alpha helical content in hFADS6 was estimated at 39\%, comparable to $32 \%$ in hFADS2. 
The presence and thermal stability of secondary structure elements was assessed on either protein through temperature ramp experiments carried out by progressively heating protein solutions from $20^{\circ} \mathrm{C}$ to $95^{\circ} \mathrm{C}$ at $1{ }^{\circ} \mathrm{C} \cdot \mathrm{min}^{-1}$. In these experiments, both proteins gave a well-defined transition between the structured and the denatured state, confirming that even the shorter protein construct in hFADS6 has a defined structure. Tm values for the transition to denatured state in hFADS6 was $51.6{ }^{\circ} \mathrm{C}$, slightly lower than the Tm measured for hFADS2 $\left(53.4^{\circ} \mathrm{C}\right)$. Whether the lower thermal stability of hFADS6 with respect to hFADS2 depends on the absence of structure-stabilizing features in the shorter isoform or on its monomeric state remains to be ascertained.
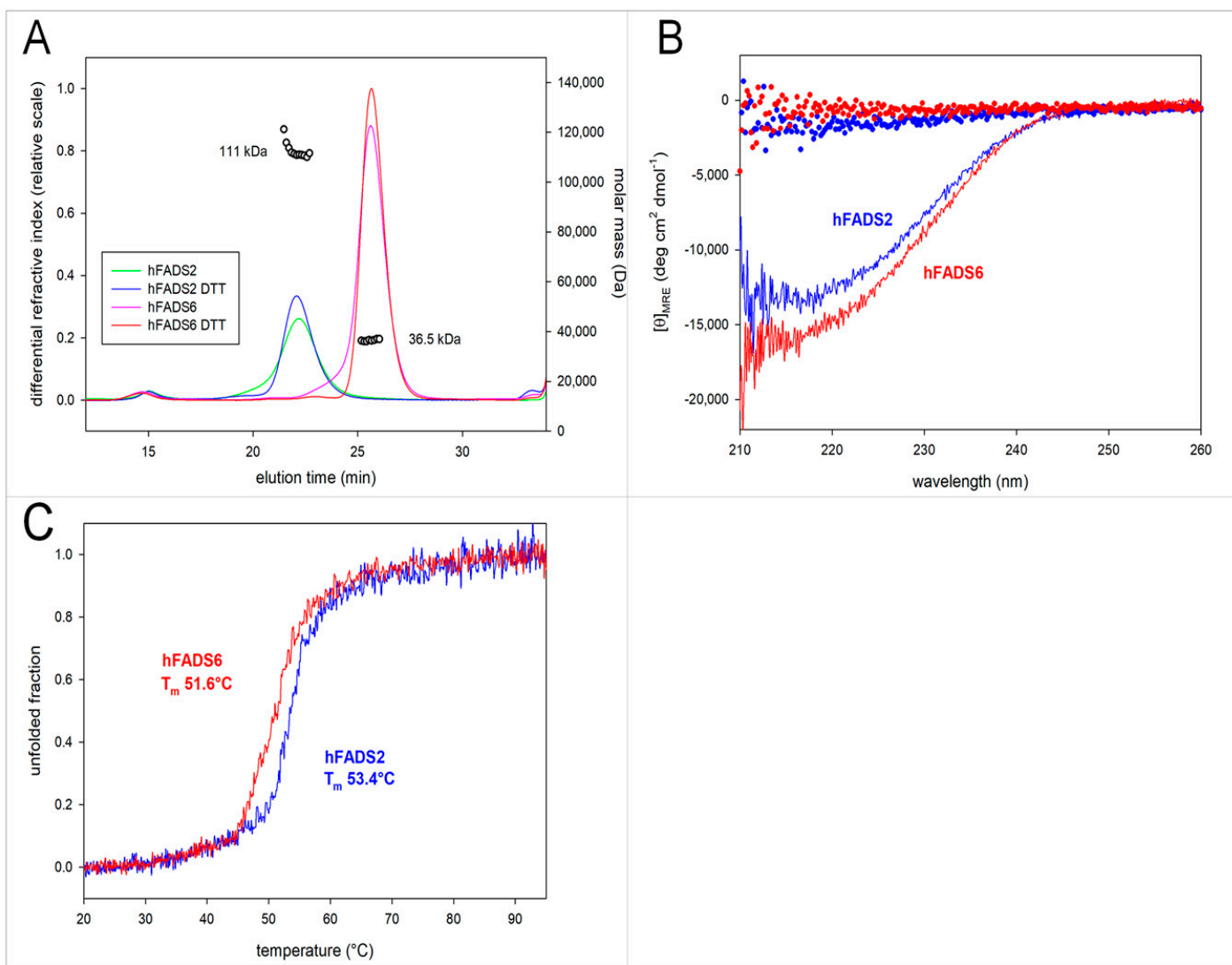

Figure 4. Characterization of the recombinant protein. (A) Tandem HPLC-GPC/LS profiles of 6-His-hFADS6 and 6-His-hFADS2. Proteins were separated on a Superose 12 column, connected in series to a dual wavelength detector, a refractive index detector, and a Dawn Heleos MALS system. The column was run in $40 \mathrm{mM}$ Hepes/Na, $150 \mathrm{mM} \mathrm{NaCl}, \mathrm{pH} 7.4$, containing $1 \mathrm{mM}$ DTT when indicated. Empty circles above individual protein peaks indicate the molecular mass calculated from MALS data; (B) Far-UV circular dichroism spectra of 6-His-hFADS6 and 6-His-hFADS2 in the absence (full lines) and in the presence (dotted lines) of $6 \mathrm{M}$ urea. Spectra were recorded at $20{ }^{\circ} \mathrm{C}$ in $1-\mathrm{mm}$ path length cells, on $0.2 \mathrm{mg} \cdot \mathrm{mL}^{-1}$ protein solutions in $40 \mathrm{mM}$ Hepes $/ \mathrm{Na}, 150 \mathrm{mM} \mathrm{NaCL}, 1 \mathrm{mM}$ DTT, $\mathrm{pH} 7.4$; (C) Temperature ramp experiments were performed on $0.2 \mathrm{mg} \cdot \mathrm{mL}^{-1}$ protein solutions in $40 \mathrm{mM}$ Hepes/Na, $150 \mathrm{mM} \mathrm{NaCl}, 1 \mathrm{mM}$ DTT, $\mathrm{pH} 7.4$ by increasing the temperature from $20{ }^{\circ} \mathrm{C}$ to $95^{\circ} \mathrm{C}$ at a heating rate of $1{ }^{\circ} \mathrm{C}$ min while continuously monitoring the loss of $\mathrm{CD}$ signal at $220 \mathrm{~nm}$. Both FADS isoforms show a cooperative folding/unfolding transition. Data were processed to calculate the midpoint temperature $\left(\mathrm{T}_{\mathrm{m}}\right)$ for the native/denatured transition $\left(53.4{ }^{\circ} \mathrm{C}\right.$ for hFADS2 vs. $51.6{ }^{\circ} \mathrm{C}$ for hFADS6).

\subsection{Functional Characterization of hFADS6 Recombinant Protein}

To ascertain if the over-expressed recombinant hFADS6 protein was able to synthesize FAD, the purified protein was incubated with the substrates, in the absence or presence of $\mathrm{Mg}^{2+}$, which is 
specifically required by other human FADS isoforms [21,22]. The FAD synthesis reaction (Figure 5A) was started by the addition of $0.13 \mu \mathrm{M}$ purified hFADS6 to ATP $(100 \mu \mathrm{M})$ and FMN $(2 \mu \mathrm{M})$. An evident fluorescence decrease ( $\lambda$ excitation at $450 \mathrm{~nm}, \lambda$ emission at $520 \mathrm{~nm}$ ) was observed, indicating the conversion of the highly fluorescent FMN into the less fluorescent FAD. The initial rate of FAD synthesis in the cuvette, calculated as described in Materials and Methods, was about $0.49 \mathrm{nmol} \cdot \mathrm{min}^{-1}$ (corresponding to $2.3 \mathrm{~min}^{-1}$ in this preparation, $2.7 \pm 0.13 \mathrm{~min}^{-1}$ as the mean of three different protein preparations.) As expected for FADSs, in the absence of $\mathrm{Mg}^{2+}$, nearly no fluorescence decrease was observed [21,22].

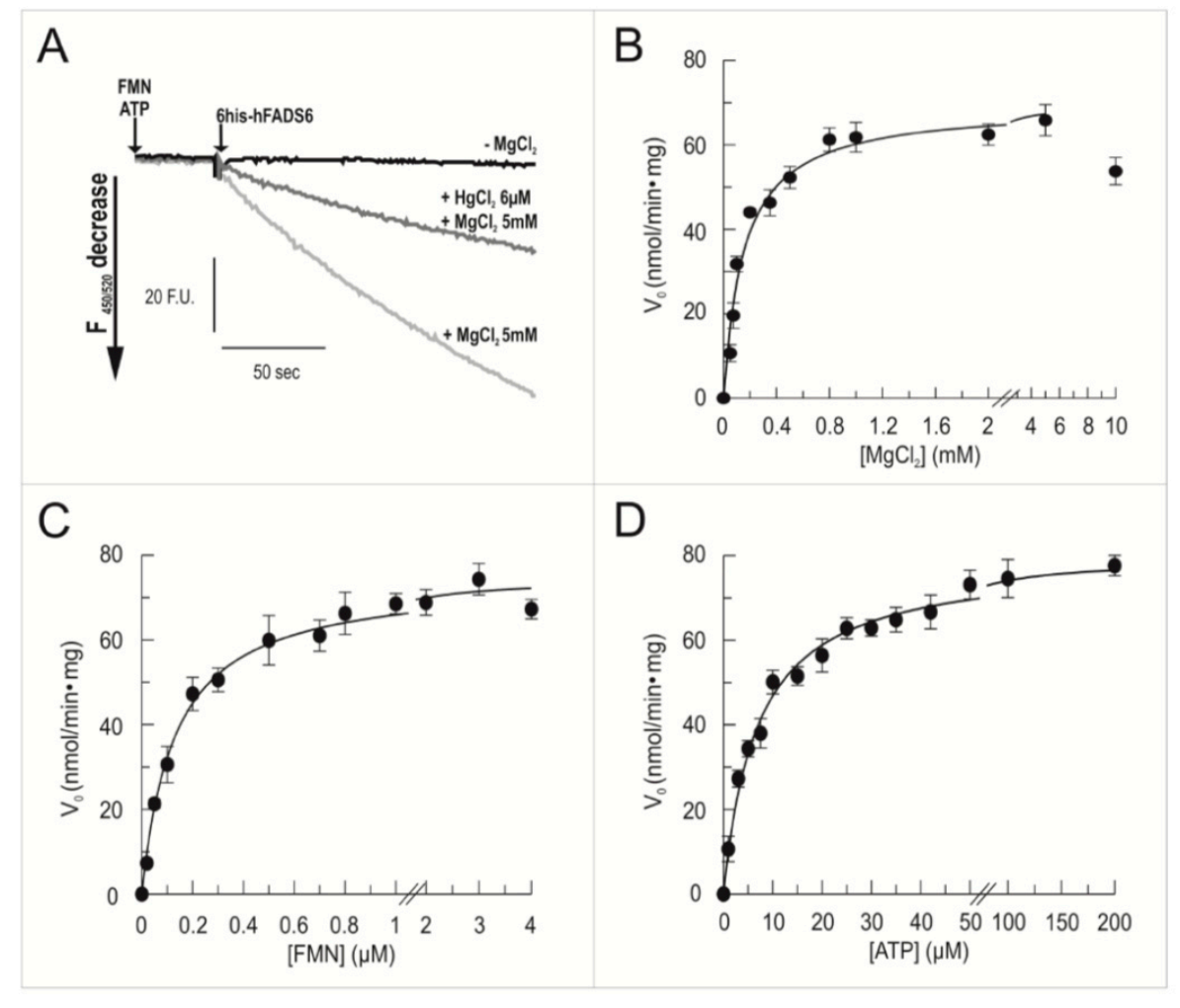

Figure 5. Fluorimetric evidence of FAD synthesis. (A) FAD synthesis was followed in $2 \mathrm{~mL}$ of $50 \mathrm{mM}$ Tris/ $\mathrm{HCl}$, pH 7.5, containing $2 \mu \mathrm{M}$ FMN and $100 \mu \mathrm{M}$ ATP, at $37^{\circ} \mathrm{C}$, in the absence of $\mathrm{MgCl}_{2}(\mathrm{black}$ line) and in the presence of $5 \mathrm{mM} \mathrm{MgCl}_{2}$ (light grey) or in the presence of $5 \mathrm{mM}$ of $\mathrm{MgCl}_{2}$ plus $6 \mu \mathrm{M}$ $\mathrm{HgCl}_{2}$ (dark grey). The FAD synthesis reaction was started by the addition of 6 His-hFADS6 $(0.11 \mu \mathrm{M})$ and measured by the initial rate of fluorescence decrease $(\lambda$ excitation $=450 \mathrm{~nm}, \lambda$ emission $=520 \mathrm{~nm})$; (B) $\mathrm{MgCl}_{2}$ concentration dependence. FAD synthesis rate, catalyzed by purified 6 His-hFADS6 $(0.13 \mu \mathrm{M})$,

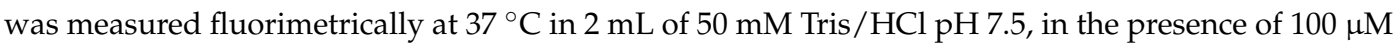
ATP, $2 \mu \mathrm{M}$ FMN, and of the given $\mathrm{MgCl}_{2}$ concentrations; (C) FMN concentration dependence. FAD synthesis rate, catalyzed by purified 6-His-hFADS6 $(0.13 \mu \mathrm{M})$ was measured fluorimetrically at $37^{\circ} \mathrm{C}$ in $2 \mathrm{~mL}$ of $50 \mathrm{mM}$ Tris/ $\mathrm{HCl} \mathrm{pH} 7.5$, in the presence of $5 \mathrm{mM} \mathrm{MgCl}_{2}, 100 \mu \mathrm{M}$ ATP, and of the given FMN concentrations; (D) ATP concentration dependence. FAD synthesis rate, catalyzed by purified 6-His-hFADS6 $(0.13 \mu \mathrm{M})$, was measured fluorimetrically at $37^{\circ} \mathrm{C}$ in $2 \mathrm{~mL}$ of $50 \mathrm{mM}$ Tris $/ \mathrm{HCl} \mathrm{pH} 7.5$, in the presence of $5 \mathrm{mM} \mathrm{MgCl} 2,2 \mu \mathrm{M} F M N$ and of the given ATP concentrations.

A relevant feature of hFADS2 is the presence of redox reactive cysteines, some of which implicated in the catalytic activity [26]. To directly evaluate if some Cys residues of hFADS6 play a role in enzyme function, the effect of $\mathrm{HgCl}_{2}$ was tested. Addition of $\mathrm{HgCl}_{2}$ inhibited the FAD synthesis reaction, in agreement with previous data described for the truncated form of FADS missing the N-terminal domain [26]. This inhibitory effect corroborates the proposal that free Cys residues located in the PAPS domain participate in the catalytic cycle of the hFADSs. 
Kinetic analyses of FAD synthesis as function of $\mathrm{MgCl}_{2}$ (Figure 5B), $\mathrm{FMN}$ (Figure 5C) or adenosine triphosphate (ATP) (Figure 5D) concentration were performed. A hyperbolic dependence was found for FAD synthetic rate on $\mathrm{Mg}^{2+}$ concentration, with $50 \%$ of maximum activity $\left(\mathrm{Mg}^{2+}{ }_{50}\right)$ at $0.15 \mathrm{mM} \pm 0.02 \mathrm{mM}$, in line with hFADS2 values [25]. Data fitting according to the Michaelis-Menten equation using the Grafit program (see Material and methods) gave apparent values of: $\mathrm{K}_{\mathrm{m}} \mathrm{FMN}$ $0.13 \pm 0.01 \mu \mathrm{M}$ and $\mathrm{K}_{\mathrm{m}}$ ATP $6.91 \pm 0.51 \mu \mathrm{M}$ (data from 3 independent experiments). Overlapping results were obtained by interpolating the same data in the Lineweaver-Burk equation using the Grafit program (not shown). These experiments gave $\mathrm{k}_{\text {cat }}$ values of 2.8 and $3.0 \mathrm{~min}^{-1}$, respectively. In three different experiments a mean value of $2.9 \pm 0.05 \mathrm{~min}^{-1}$ was evaluated.

The reverse reaction, i.e., pyrophosphorolysis, was also tested, as well as the ability to cleave FAD by hydrolysis, as previously reported for the bi-functional isoform 2 [28]. As expected on the basis of the lack of the MPTb domain, isoform 6 of the enzyme was able to catalyze the pyrophosphorolysis, but not the hydrolysis (Figure 6A); not even the presence of $\mathrm{Co}^{2+}$ could promote the ability of hFADS6 to catalyze hydrolysis, at a difference from the bi-functional variant. A control was also made by using the -SH reagent mersalyl. Mersalyl induces FAD hydrolysis in the hFADS2 isoform, but was found inactive in this regard with the hFADS6 variant. The kinetics of the reverse reaction was measured as a function of NaPPi concentration (Figure 6B). A $\mathrm{K}_{\mathrm{m}}$ PPI of $0.042 \pm 0.006 \mathrm{mM}$ was calculated by interpolating the data in the Michaelis-Menten equation. The $\mathrm{K}_{\mathrm{m}} \mathrm{FAD}$ could not be measured due to instrumental limitations that do not allow measurements of $K_{m}$ values lower than $0.1 \mu \mathrm{M}$ as in the case hFADS6, similarly to hFADS2 [25].

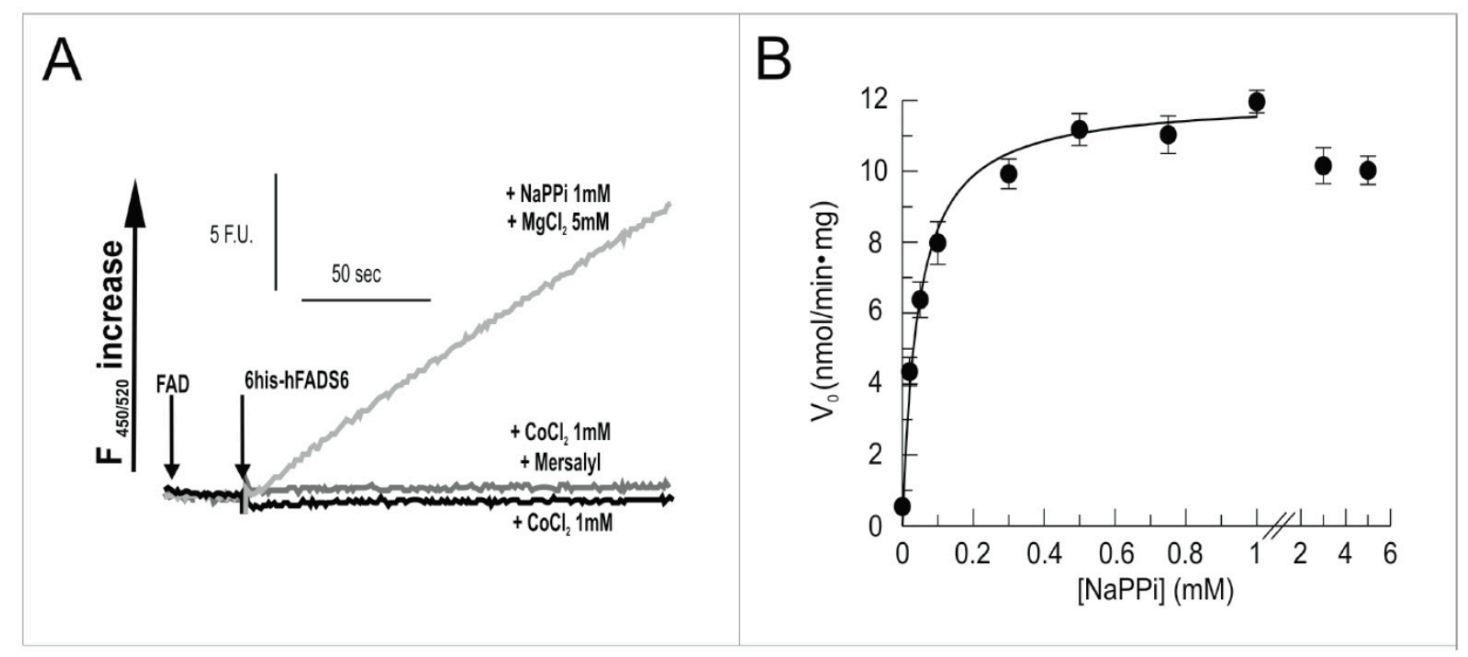

Figure 6. Fluorimetric evidence for FAD cleavage as catalyzed by 6-His-hFADS6. (A) Fluorimetric evidence of FAD cleavage. The reaction was measured in $50 \mathrm{mM}$ Tris $/ \mathrm{HCl}, \mathrm{pH} 7.5$, containing $0.5 \mu \mathrm{M}$ FAD and $1 \mathrm{mM} \mathrm{NaPPi}$, at $37^{\circ} \mathrm{C}$, in the presence of $5 \mathrm{mM} \mathrm{MgCl}_{2}$ (light grey). The FAD cleavage reaction (i.e., pyrophosporolysis) was started by the addition of the 6-His-hFADS6 protein $(0.13 \mu \mathrm{M})$ and was measured by the initial rate of fluorescence increase $(\lambda$ excitation $=450 \mathrm{~nm}, \lambda$ emission $=520 \mathrm{~nm})$. FAD hydrolysis was measured under the same conditions, if not for omitting $\mathrm{NaPPi}$ and $\mathrm{MgCl}_{2}$, and in the presence of either $1 \mathrm{mM} \mathrm{CoCl}_{2}$ (black line) or $1 \mathrm{mM} \mathrm{CoCl}_{2}$ plus $0.5 \mathrm{mM}$ Mersalyl (dark grey); (B) NaPPi concentration dependence. FAD cleavage (i.e., pyrophosporolysis) rate, catalyzed by purified 6-His-hFADS6 $(0.13 \mu \mathrm{M})$, was measured fluorimetrically at $37^{\circ} \mathrm{C}$ in $2 \mathrm{~mL}$ of $50 \mathrm{mM}$ Tris $/ \mathrm{HCl} \mathrm{pH} 7.5$, in the presence of $5 \mathrm{mM} \mathrm{MgCl}_{2}, 0.5 \mu \mathrm{M} F A D$, and of the given NaPPi concentrations.

\subsection{D Model of hFADS6}

Structural models of hFADS6 apo-protein were computed with three different programs, using homology and/or threading procedures. The C-terminus part of hFADS6 (PAPS domain) is homologous to already experimentally characterized proteins, with identity/similarity levels 
adequate for successfully producing homology models with an accurate architecture: for instance, query primary structure (aa 109-320) has a 36\% similarity with Saccharomyces cerevisiae YDL045c FAD synthetase. Accordingly, the output from the three different modeling approaches was very similar when predicting by homology modeling the organization of the C-terminus part of hFADS6. On the contrary, the produced models largely diverged in the arrangement of the N-terminus part, for which no homologous templates can be identified. For this reason, Swiss Model, which is a software exclusively based on homology modeling, failed in predicting 3D structure of the hFADS6 N-terminus. Robetta or I-TASSER, which also implement ab initio modeling, produced dissimilar 3D structures for this part. The output provided by Robetta was selected as final apo-hFADS6 model, for its better geometrical features. FAD ligand was transferred, after superposition with the crystallographic structure of the Candida glabrata FMN Adenylyltransferase (PDB ID: 3g6k) for generating the holo-hFADS6 form (Figure 7, PAPS domain in white, N-terminus part in red). In more general terms, the predicted model for hFADS6 had an alpha-helical content of 37\% (117 residues out of 320), close to that calculated from CD spectra (39\%). The content of beta-strands cannot be determined by the limited coverage of our CD spectra, but it was estimated at $10 \%$ in the model (32 residues out of 320 ).

A

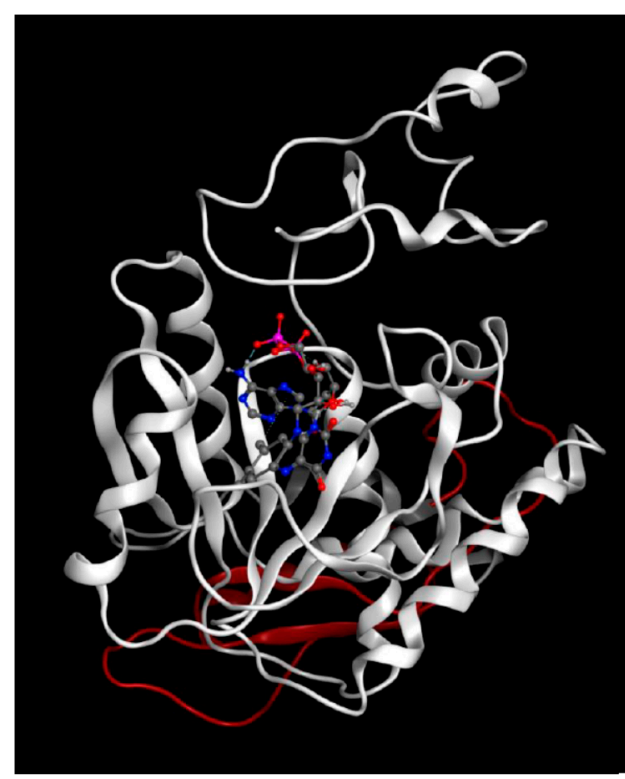

B

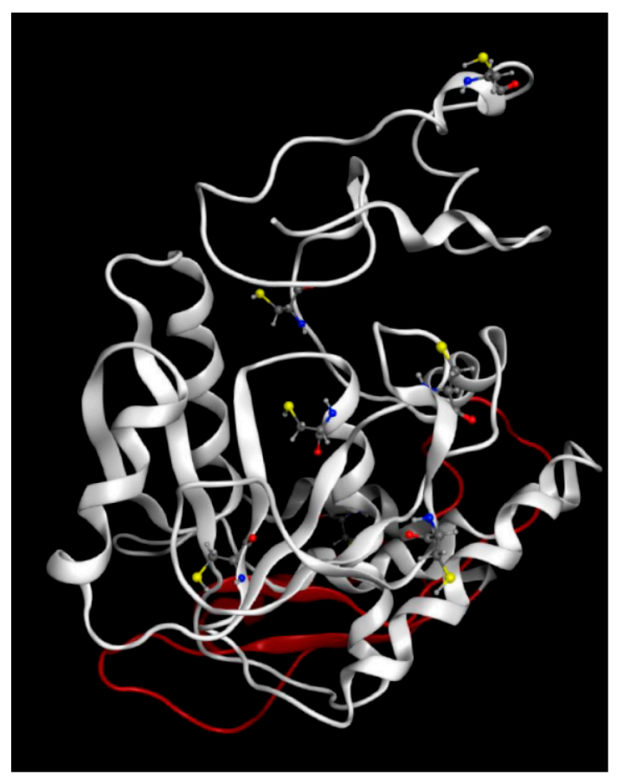

Figure 7. Three-dimensional (3D) model of hFADS6. Ribbon representation of hFADS6 modeled through Robetta as described in Materials and Methods. (A) FAD has been transferred in the active site from PDB ID: $3 g 6 \mathrm{k}$ and is represented in ball and stick. The PAPS reductase domain is colored in white, the N-terminus part of hFADS6 is colored in red. (B) Cys residues are represented in ball and stick and colored by elements. Element colors in (A,B): Sulfur, yellow; Nitrogen, blue; Oxygen, red; Phosphorus, pink; Carbon, grey.

\section{Discussion}

The FADS6 isoform was very recently discovered, by transcriptome analysis, in patients harboring frameshift mutations of FLAD1 gene triggering RR-MADD. This FADS6 isoform can be anyway produced from the altered gene since it starts from Met268 whose codon is downstream the frameshift ([30], and Figure 1). However, it was not known if this isoform could display some functions related to FAD production. We have demonstrated in this work that this shorter isoform is able to synthesize FAD. Therefore, its importance, even though expressed in small amount, resides in human pathophysiology, that is, to synthesize sufficient amount of FAD, allowing survival of patients affected by disruptive mutations of the FLAD1 gene. hFADS6 harbors additional 61 N-terminus 
amino acid residues with respect to the artificial PAPS mutant. Hence, it could then be considered the natural counterpart of the artificial protein previously produced by recombinant DNA strategies [29]. Therefore, it was very important to ascertain the capacity of the novel hFADS6 isoform to synthesized FAD. FADS6 displayed FAD synthesizing activity, but not FAD hydrolase activity, which resides in the $\mathrm{N}$-terminal domain of the full length FADS1 or 2 [28]. Moreover, FADS6 showed some similar features to the other previously characterized longer FADS isoforms, such as $\mathrm{Mg}^{2+}$ dependence and strong inhibition by $\mathrm{Hg}^{2+}$, confirming that some of the Cys residues highlighted in the model (Figure 7) play crucial roles in this PAPS domain [26]. The FAD synthesizing activity is about $70 \%$ with respect to that measured for hFADS2 [25]; however, the catalytic efficiency $\left(\mathrm{k}_{\mathrm{cat}} / \mathrm{K}_{\mathrm{m}}\right)$ results higher than that of hFADS2 due to the lower Km values of hFADS6 for both FMN and ATP compared to those of hFADS2. The data are in agreement with those found for PAPS [29]. Concerning the $\mathrm{Mg}^{2+}$ binding, we expect a similar behavior as in C. glabrata FMN Adenylyltransferase, i.e., $\mathrm{Mg}^{2+}$ makes a bridge between bound ATP and the protein binding site. In fact, hFADS6 harbors two residues, D141 and D225, which are homologous to those responsible for $\mathrm{Mg}^{2+}$ binding in C. glabrata enzyme (D66 and D168) [34].

From a structural standpoint, it is noteworthy that the short and monomeric hFADS6 appears to be appropriately folded, with a thermal stability of its folding remarkably close to that of the much larger (and dimeric) hFADS2. The presence of DTT has effect neither on the aggregation state nor on the hydrodynamic radius of the two proteins, confirming the absence of structurally relevant disulfide bonds in the structure.

In agreement with the high sequence identity between the two primary structures, the models for the 3D structure of the C-terminus part of hFADS6 and of the PAPS mutant previously produced through recombinant DNA are similar to one another (Figure 7, white structure), except for the N-terminal 61 amino acids of the hFADS6 (Figure 7, red structure). Besides the overall close similarity of the fold, even the positions of the Cys residues in the two structures mostly overlap [29]. Thanks to the availability of a number of templates with similarity with the target higher than $30 \%$, the prediction of the general architecture of this part of hFADS6 can be considered accurate. Conversely, lack of adequate templates forces to rely on threading and ab initio modeling for the $\mathrm{N}$-terminus part of hFADS6, accepting a lower confidence of the outcome. Indeed, the results provided by Robetta and I-TASSER for the N-terminus of the hFADS6 diverge for their overall orientation with respect to the PAPS domain. In neither case, however, the access to the catalytic site is hampered by the N-terminus part of the protein. Similarity of the 3D arrangement of these two isoforms in the C-terminus part is the structural basis for a conserved catalytic activity. Indeed, docking of FAD on hFADS6 (Figure 7A) led to similar results with respect to the PAPS [29].

Despite the hFADS6 synthase function overlaps that of hFADS2, clear structural differences were observed between the two proteins. Indeed, differently from the dimeric structure of hFADS2, hFADS6 had an unequivocal monomeric form. Since the major difference in hFADS6 with respect to hFADS2 is the lack of the molybdopterin binding domain, this difference could be the cause of the absence of the dimeric structure. In other words, in hFADS2, the molybdopterin binding domain should play some role in the interaction between the two monomers. Indeed, previous studies based on site-directed mutagenesis addressed the relationships between the molybdopterin binding and the PAPS domains [26]. Further conditioning of the structure determined by the redox state (SH/S-S balance) of the protein is likely to occur; a crucial role for some Cys residues in the PAPS domain of the hFADS2 was assessed by site-directed mutagenesis. This issue is under investigation for hFADS6 in comparison with the hFADS2 isoform.

The first evidence of the expression of hFADS6 isoform in human tissues was provided in 2016 [30]. However, a clear correlation on expression level in healthy and pathological conditions is still lacking. Another interesting question concerns the putative localization of this novel isoform. Since this hFADS6 lacks the mitochondrial targeting peptide [27] a sole cytosolic localization is expected. This point is particularly relevant for cellular bioenergetics. Indeed, the relative activity of mitochondrial vs. cytosolic isoforms of FADS might regulate the transport directionality of the mitochondrial FAD 
transporter [35]. Therefore, under the conditions in which frameshift mutations of FLAD1 gene alter the relationships among splice variants, this novel emergency protein becomes relevant and we expect that flavoprotein biogenesis in mitochondria is ensured by FAD import. Experiments are on the way to directly prove these assumptions in the frame of measuring the relative abundance of the natural hFADS6 in different tissues.

In conclusion, FADS6 represents a previously neglected FADS isoform whose role was highlighted by the discovery of FADS gene natural mutations causative of RR-MADD [30]. Very importantly, this isoform may represent an emergency protein, which can be produced also in patients from an altered gene. In this respect, this protein represents a target for therapy intervention in patients harboring FADS defects, especially for those in which Rf therapy is unsuccessful.

\section{Materials and Methods}

\subsection{Materials}

All chemicals and monoclonal anti-polyhistidine-peroxidase antibody (A7058) were from Sigma-Aldrich (Saint Louis, MO, USA), if not otherwise specified. E. coli Rosetta(DE3)pLysS strain was purchased from Novagen (Madison, WI, USA). Restriction endonucleases and other cloning reagents were purchased from Fermentas (Burlington, ON, Canada); MegaMan Human Transcriptome Library was from Agilent Technologies (Santa Clara, CA, USA). Anti-hFADSs antibody (patent number WO2009107158 A1) was in-house produced.

\subsection{Cloning of cDNA Coding for hFADS6}

The $963 \mathrm{bp}$ cDNA coding for the hFADS6 isoform [30] was amplified from the Mega Man Human Transcriptome Library with the forward and reverse primers $5^{\prime}$-CCGGAATTCTATGAAG GGACTATTCCAAAACCCAG-3' and 5'-CCGCTCGAGCTACCCCTGCTGTCCTGGGAAGGGG-3', containing the EcoRI and XhoI sites, respectively. The amplified cDNA was then cloned in the EcoRI/XhoI sites of the pH6EX3 expression vector. The resulting recombinant plasmid, defined as pH6EX3-hFADS6, encodes a 6His-tagged fusion protein corresponding to hFADS6 carrying the extra N-terminal sequence MSPIHHHHHHLVPRGSEASNS.

\subsection{Expression of Recombinant hFADS6 Protein in E. coli}

The expression host E. coli Rosetta(DE3)pLysS strain was transformed with the pH6EX3-hFADS6 construct by calcium chloride treatment. Selection of positive colonies was performed on LB-agar plates containing $34 \mu \mathrm{g} \cdot \mathrm{mL}^{-1}$ chloramphenicol and $100 \mu \mathrm{g} \cdot \mathrm{mL}^{-1}$ ampicillin. E. coli Rosetta(DE3)pLysS cells carrying the recombinant plasmid were inoculated in $10 \mathrm{~mL}$ of LB medium (1\% Bacto Peptone, $0.5 \%$ Bacto Yeast extract, $1 \% \mathrm{NaCl}$, pH 7.0) supplemented with $100 \mu \mathrm{g} \cdot \mathrm{mL}^{-1}$ ampicillin and $34 \mu \mathrm{g} \cdot \mathrm{mL}^{-1}$ chloramphenicol, and cultured overnight at $37^{\circ} \mathrm{C}$ with rotary shaking $(160 \mathrm{rpm})$. A $5 \mathrm{~mL}$ aliquot of the cell culture was transferred to $0.5 \mathrm{~L}$ of fresh $\mathrm{LB}$ medium supplemented with $100 \mu \mathrm{g} \cdot \mathrm{mL}^{-1} \mathrm{ampicillin}$ and $34 \mu \mathrm{g} \cdot \mathrm{mL}^{-1}$ chloramphenicol and grown at $37^{\circ} \mathrm{C}$ to $\mathrm{A}_{600}$ equal to $0.6-0.8$. Then, $0.4 \mathrm{mM}$ IPTG was added to induce the expression of the recombinant protein, 6-His-hFADS6. Growth was continued for 2 to $8 \mathrm{~h}$ at $28^{\circ} \mathrm{C}$, or overnight at $20^{\circ} \mathrm{C}$. Bacteria were harvested by centrifugation at $3000 \times g$ for $10 \mathrm{~min}$ at $4{ }^{\circ} \mathrm{C}$ and the pellets stored at $-20^{\circ} \mathrm{C}$. The bacterial pellet (about $3 \mathrm{~g}$ wet weight) was thawed on ice for $15 \mathrm{~min}$ and resuspended in $30 \mathrm{~mL}$ of start buffer (500 mM NaCl, $40 \mathrm{mM}$ Hepes/Na, pH 7.4) supplemented with Protease Inhibitor Cocktail (P8849, Sigma-Aldrich, $1 \mathrm{~mL} / 20 \mathrm{~g}$ of cells wet weight) and $0.5 \mathrm{mM}$ Phenylmethylsulfonyl fluoride (PMSF). Cells were disrupted by mild sonication at $4{ }^{\circ} \mathrm{C}$ (one cycle of $10 \mathrm{~min}$ and one cycle of $5 \mathrm{~min}$ with $1 \mathrm{~s}$ Pulse ON and $1 \mathrm{~s}$ Pulse OFF, at $40 \mathrm{~W}$ ) using a VCX130 PB sonifier (Branson, Danbury, CT, USA). The soluble and the insoluble cell fractions were separated by centrifugation of the cell lysate at $20,000 \times \mathrm{g}$ for $30 \mathrm{~min}$ at $4{ }^{\circ} \mathrm{C}$. The pellet (inclusion bodies and cell debris), containing the insoluble over-expressed proteins, was re-suspended in $15 \mathrm{~mL}$ of start buffer, aliquoted and used for SDS-PAGE analysis. The supernatant, containing the soluble 
over-expressed 6-His-hFADS6, was used for SDS-PAGE analysis, FADS activity assay, and further protein purification (see below).

\subsection{Purification of Recombinant 6-His-hFADS6}

A $40 \mathrm{~mL}$ aliquot of the soluble cell fraction, obtained as outlined above, was applied onto a Chelating Sepharose Fast Flow column ( $3.5 \mathrm{~mL}$ packed resin), previously treated with $200 \mathrm{mM} \mathrm{NiSO}_{4}$ according to the producer's protocol and equilibrated with the start buffer. The column was first washed with $35 \mathrm{~mL}$ start buffer, then eluted with a step gradient of 50,100, 250, and $500 \mathrm{mmol} \cdot \mathrm{L}^{-1}$ imidazole in the same buffer. Prior to storing or further processing, fractions containing the purified recombinant protein were desalted by gel filtration on a PD10-column in $40 \mathrm{mM}$ Hepes/ $\mathrm{Na}, 5 \mathrm{mM}$ $\beta$-mercaptoethanol, $\mathrm{pH}$ 7.4. These protein samples were stable for at least 30 days at $4{ }^{\circ} \mathrm{C}$.

\subsection{Protein Concentration and FAD/Protein Monomer Ratio Measurements}

Protein concentration was measured with the method of Bradford, using BSA as standard [36]. In an alternative procedure, protein concentration of the purified 6-His-hFADS6 was estimated by absorbance spectra, which were recorded on an Ultrospec 3100 pro spectrophotometer (Amersham Biosciences, Piscataway, NJ, USA), essentially as in [25]. To this aim, the contribution of the bound FAD had to be subtracted from the $A_{280}$ readings. Because $A_{280}$ for both free FAD and hFADS6-bound FAD is 1.7-fold $A_{450}$, the $A_{280}$, actually due to the apo-protein, may be calculated from the Equation:

$$
\mathrm{A}_{280} \text { apo-enzyme }=\mathrm{A}_{280}-\left(\mathrm{A}_{450} \times 1.7\right)
$$

The protein concentration was then estimated by using $\varepsilon_{280}\left(47.705 \mathrm{mM}^{-11} \cdot \mathrm{cm}^{-1}\right.$, $1.247 \mathrm{mg} / \mathrm{mL}^{-1} \cdot \mathrm{cm}^{-1}$ ), as calculated from the protein sequence by using the ExpasyProtParam tool (Swiss Institute of Bioinformatics, Lausanne, Switzerland). Measurements made by either the spectrophotometric or the Bradford method differed by no more than $7 \%$. The FAD/protein monomer ratio (given as percent flavinylation, $\mathrm{Fl} \%$ ) could be estimated from the absorbance spectrum by considering:

$$
\mathrm{Fl} \%=\left[\left(\mathrm{A}_{450} / \mathrm{A}_{280} \text { apo-enzyme }\right) / 0.23\right] \times 100
$$

where 0.23 is the $\varepsilon_{450}(\mathrm{FAD}) / \varepsilon_{280}$ (apo-enzyme) ratio.

\subsection{FAD Content as Assayed by HPLC Analysis}

The purified 6-His-hFADS6 (15.4 $\mu \mathrm{g}$ in $100 \mu \mathrm{L}, 4 \mu \mathrm{M})$ was treated with $10 \%$ perchloric acid to remove tightly but not covalently bound molecules. The supernatant was neutralized and aliquots were analyzed for flavin content by HPLC, as previously reported [25,37]. Standard aqueous of FAD, FMN and riboflavin were used to identify the protein-bound flavin cofactor.

\subsection{Circular Dichroism Measurements}

Circular dichroism (CD) spectra were recorded at $20^{\circ} \mathrm{C}$ in $0.1 \mathrm{~cm}$ path length cells on $0.2 \mathrm{mg} \cdot \mathrm{mL}^{-1}$ protein solutions in $40 \mathrm{mM}$ Hepes/Na, $150 \mathrm{mM} \mathrm{NaCl}, 1 \mathrm{mM}$ DTT, pH 7.4 by means of a J810 spectropolarimeter (Jasco Europe SrL, Cremella, Italy), and were analyzed by means of the J800 software (Jasco Europe SrL, Cremella, Italy). Similar conditions were used for temperature ramp experiments. Temperature was increased from 20 to $95^{\circ} \mathrm{C}$ at a heating rate of $1{ }^{\circ} \mathrm{C} \cdot \mathrm{min}^{-1}$ by means of a computer-controlled Peltier-driven cell holder, while continuously monitoring the loss of CD signal at $220 \mathrm{~nm}$. CD spectra were normalized in terms of Mean Residual Ellipticity ([$\left[\Theta_{\mathrm{MRE}},[38]\right)$, and the alpha-helical content was estimated from the [$\Theta_{\text {MRE }}$ at $222 \mathrm{~nm}$ according to Chen and Yang [39]. 


\subsection{Molecular Parameters}

The molecular size of the purified 6-His-hFADS6 was assessed by HPLC-GPC, using a Superose 12 column (10/300 GL, GE Healthcare, Milan Italy), fitted to a HPLC system (mod. 515) equipped with a tunable dual wavelength detector (Waters 2487, Milford, MA, USA) and a refractive index detector $\left(\right.$ Optilab $^{\circledR}$ T-rEX, Wyatt, Santa Barbara, CA, USA). Columns were run at $0.5 \mathrm{~mL} \cdot \mathrm{min}^{-1}$ in $40 \mathrm{mM}$ Hepes/Na, $150 \mathrm{mM} \mathrm{NaCl}$, pH 7.4, containing $1 \mathrm{mM}$ DTT when indicated, and calibrated with suitable protein standards that encompassed the molecular size predicted for hFADS2. The hydrodynamic properties and the molar mass of the purified 6-His-hFADS6 were assessed by Multi Angle Light Scattering (MALS), using a Wyatt Dawn ${ }^{\circledR}$ Heleos ${ }^{\circledR}$ system connected in series to the aforementioned HPLC-GPC chromatographic system. Molar mass at different elution times was calculated by means of the Astra software (Wyatt, Santa Barbara, CA, USA), using a dn/dc value of 0.185.

\subsection{Measurements of FAD Synthesis and FAD Pyrophosphorolysis Rate}

The rate of FAD synthesis and FAD cleavage were measured as in [25], by exploiting the different fluorescence properties of FAD with respect to FMN. Fluorescence time courses ( $\lambda$ excitation at $450 \mathrm{~nm}$; $\lambda$ emission at $520 \mathrm{~nm}$ ) were followed at $37^{\circ} \mathrm{C}$ in a FP-3800 spectrofluorometer (Jasco, Easton, MD, USA). In each experiment, FAD and FMN fluorescence were calibrated by using standard solutions. The rate of FAD synthesis, expressed as nmol FAD $\mathrm{min}^{-1} \cdot(\mathrm{mg} \text { protein })^{-1}$, was calculated from the rate of fluorescence decrease, measured as the tangent to the initial part of the experimental curve, as described in detail in [25]. For activity measurements, purified protein fractions (5-10 $\mu \mathrm{g}, 0.13-0.26 \mathrm{nmol}$ protein

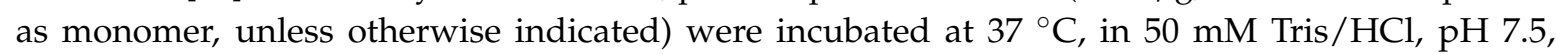
containing $5 \mathrm{mM} \mathrm{MgCl}_{2}, 2 \mu \mathrm{M} \mathrm{FMN}, 100 \mu \mathrm{M}$ ATP, and additional reagents as appropriate.

The rate of FAD pyrophosphorolysis was measured in the presence of $1 \mathrm{mM} \mathrm{NaPPi}$ (sodium pyrophosphate) and $0.5 \mu \mathrm{M}$ FAD as in [25]. The rate of FAD cleavage was expressed as nmol FAD $\min ^{-1} \cdot(\mathrm{mg} \text { protein })^{-1}$, and was calculated from the rate of fluorescence increase, measured as the tangent to the initial part of the experimental curve. Under the same experimental conditions, but in the absence of $\mathrm{NaPPi}$, the possible occurrence of FAD hydrolysis was investigated essentially as in $[28,40]$, in the presence of $1 \mathrm{mM} \mathrm{CoCl}_{2}$.

To fit the experimental data and to obtain estimates of the kinetic parameters, the Grafit software was used (v. 5.0.13, 2006, by R.J. Leatherbarrow, Erithacus Software, Wilmington House, UK).

\subsection{Electrophoretic Analysis}

Proteins were separated by SDS-PAGE on $12 \% \mathrm{~T}$ polyacrylamide gels, according to Laemmli [41]. Quantitative evaluation of Coomassie Blue-stained protein bands was carried out using the Chemidoc imaging system (Bio-Rad, Hercules, CA, USA) and the Quantity One software (Bio-Rad, Hercules, CA, USA), as described previously [42].

\subsection{Immunoblotting}

SDS-PAGE-separated proteins were electro-transferred onto a nitrocellulose membrane using a trans-blot semidry electrophoretic transfer cell (Eppendorf, Hamburg, D). The immobilized proteins were incubated $1 \mathrm{~h}$ either with a 2500-fold dilution of an in-house produced polyclonal antiserum against hFADSs or with a 12,000-fold dilution of a peroxidase-conjugated anti-polyhistidine antibody. The bound antibodies were visualized with the aid of a secondary anti-rabbit immunoglobulin antibody, conjugated with alkaline phosphatase (1:5000 dilution).

\subsection{D Modeling of hFADS6}

A three-dimensional model was obtained by submitting the target sequence to three homology and/or ab initio servers: Swiss Model (https:/ / swissmodel.expasy.org), I-TASSER (https:/ / zhanglab. ccmb.med.umich.edu/I-TASSER/) and Robetta (http:/ / robetta.bakerlab.org) and by comparing their 
outputs. Swiss Model could only provide results for the C-terminal domain, whereas the threading/ab initio combined procedures implemented by the other two approaches produced almost complete models. Of the latter two, the model built by Robetta scored better in terms of protein geometry (Ramachandran plot, bond lengths, angles, dihedrals, rotamers, clashes, and contact energies), and was thus selected. FAD was transferred to it from one of the templates used by I-TASSER (namely, 3g6k); the resulting complex was then energy minimized with the Amber10:EHT forcefield and the reaction field electrostatics, by the Molecular Operating Environment v. 2016.0803.

Acknowledgments: This work was supported by funds from: PON01_00937__Modelli sperimentali Biotecnologici integrati per lo sviluppo e la selezione di molecole di interesse per la salute dell'uomo". MIUR (Italian Ministry of Education, Universities and Research) to C.I. and M.B.; "Fondi di Ateneo" Università degli Studi di Bari to M.B.M. Tolomeo was supported by a post-graduate research fellowship financed by "Fondazione Cassa di Risparmio di Puglia". The helpful collaboration of M.T. Damiano, who participated as a student in the early stages of this work, and the technical assistance of V. Giannoccaro (Università degli Studi di Bari) are gratefully acknowledged.

Author Contributions: M.G., F.V. and C.I. were involved in FADS6 cloning and expression. P.L., M.T. and M.B. were involved in protein purification and characterization. A.B., S.I. and F.B. were involved in HPLC-GPC/LS and thermal stability analysis; E.G. and I.E. were involved in modeling. All authors discussed the results and contributed to the final manuscript. M.B. wrote the manuscript, coordinated and supervised the work.

Conflicts of Interest: The authors declare no conflict of interest.

\section{References}

1. Barile, M.; Giancaspero, T.A.; Brizio, C.; Panebianco, C.; Indiveri, C.; Galluccio, M.; Vergani, L.; Eberini, I.; Gianazza, E. Biosynthesis of flavin cofactors in man: Implications in health and disease. Curr. Pharm. Des. 2013, 19, 2649-2675. [CrossRef] [PubMed]

2. Joosten, V.; van Berkel, W.J. Flavoenzymes. Curr. Opin. Chem. Biol. 2007, 11, 195-202. [CrossRef] [PubMed]

3. Lienhart, W.D.; Gudipati, V.; Macheroux, P. The human flavoproteome. Arch. Biochem. Biophys. 2013, 535, 150-162. [CrossRef] [PubMed]

4. McCormick, D.B. A trail of research on cofactors: An odyssey with friends. J. Nutr. 2000, 130 (Suppl. 2S), 323S-330S. [PubMed]

5. Heikal, A.A. Intracellular coenzymes as natural biomarkers for metabolic activities and mitochondrial anomalies. Biomark. Med. 2010, 4, 241-263. [CrossRef] [PubMed]

6. Finnegan, S.; Yuan, H.; Wang, Y.F.; Orville, A.M.; Weber, I.T.; Gadda, G. Structural and kinetic studies on the Ser101Ala variant of choline oxidase: Catalysis by compromise. Arch. Biochem. Biophys. 2010, 501, 207-213. [CrossRef] [PubMed]

7. Cornelius, N.; Corydon, T.J.; Gregersen, N.; Olsen, R.K. Cellular consequences of oxidative stress in riboflavin responsive multiple acyl-CoA dehydrogenation deficiency patient fibroblasts. Hum. Mol. Genet. 2014, 23, 4285-4301. [CrossRef] [PubMed]

8. Zhang, J.; Frerman, F.E.; Kim, J.J. Structure of electron transfer flavoprotein-ubiquinone oxidoreductase and electron transfer to the mitochondrial ubiquinone pool. Proc. Natl. Acad. Sci. USA 2006, 103, 16212-16217. [CrossRef] [PubMed]

9. Qin, X.; Tan, Y.; Wang, L.; Wang, Z.; Wang, B.; Wen, X.; Yang, G.; Xi, Z.; Shen, Y. Structural insight into human variegate porphyria disease. FASEB J. 2011, 25, 653-664. [CrossRef] [PubMed]

10. Yonezawa, A.; Inui, K. Novel riboflavin transporter family RFVT/SLC52: Identification, nomenclature, functional characterization and genetic diseases of RFVT/SLC52. Mol. Asp. Med. 2013, 34, 693-701. [CrossRef] [PubMed]

11. Depeint, F.; Bruce, W.R.; Shangari, N.; Mehta, R.; O’Brien, P.J. Mitochondrial function and toxicity: Role of B vitamins on the one-carbon transfer pathways. Chem. Biol. Interact. 2006, 163, 113-132. [CrossRef] [PubMed]

12. Gherasim, C.G.; Zaman, U.; Raza, A.; Banerjee, R. Impeded electron transfer from a pathogenic FMN domain mutant of methionine synthase reductase and its responsiveness to flavin supplementation. Biochemistry 2008, 47, 12515-12522. [CrossRef] [PubMed] 
13. Wolthers, K.R.; Scrutton, N.S. Protein interactions in the human methionine synthase-methionine synthase reductase complex and implications for the mechanism of enzyme reactivation. Biochemistry 2007, 46, 6696-6709. [CrossRef] [PubMed]

14. Hemberger, S.; Pedrolli, D.B.; Stolz, J.; Vogl, C.; Lehmann, M.; Mack, M. RibM from Streptomyces davawensis is a riboflavin/roseoflavin transporter and may be useful for the optimization of riboflavin production strains. BMC Biotechnol. 2011, 11, 119. [CrossRef] [PubMed]

15. Garcia-Angulo, V.A. Overlapping riboflavin supply pathways in bacteria. Crit. Rev. Microbiol. 2017, 43, 196-209. [CrossRef] [PubMed]

16. LeBlanc, J.G.; Milani, C.; de Giori, G.S.; Sesma, F.; van Sinderen, D.; Ventura, M. Bacteria as vitamin suppliers to their host: A gut microbiota perspective. Curr. Opin. Biotechnol. 2013, 24, 160-168. [CrossRef] [PubMed]

17. Said, H.M. Recent advances in transport of water-soluble vitamins in organs of the digestive system: A focus on the colon and the pancreas. Am. J. Physiol. Gastrointest. Liver Physiol. 2013, 305, G601-G610. [CrossRef] [PubMed]

18. Bennett, M.J. Brown-Vialetto-Van Laere and Fazio Londe syndromes: Defects of riboflavin transport with biochemical similarities to multiple acyl-CoA dehydrogenation defects (MADD). J. Inherit. Metab. Dis. 2012, 35, 941-942. [CrossRef] [PubMed]

19. Haack, T.B.; Makowski, C.; Yao, Y.; Graf, E.; Hempel, M.; Wieland, T.; Tauer, U.; Ahting, U.; Mayr, J.A.; Freisinger, P.; et al. Impaired riboflavin transport due to missense mutations in SLC52A2 causes Brown-Vialetto-Van Laere syndrome. J. Inherit. Metab. Dis. 2012, 35, 943-948. [CrossRef] [PubMed]

20. Bosch, A.M.; Abeling, N.G.; Ijlst, L.; Knoester, H.; van der Pol, W.L.; Stroomer, A.E.; Wanders, R.J.; Visser, G.; Wijburg, F.A.; Duran, M.; et al. Brown-Vialetto-Van Laere and Fazio Londe syndrome is associated with a riboflavin transporter defect mimicking mild MADD: A new inborn error of metabolism with potential treatment. J. Inherit. Metab. Dis. 2011, 34, 159-164. [CrossRef] [PubMed]

21. Brizio, C.; Galluccio, M.; Wait, R.; Torchetti, E.M.; Bafunno, V.; Accardi, R.; Gianazza, E.; Indiveri, C.; Barile, M. Over-expression in Escherichia coli and characterization of two recombinant isoforms of human FAD synthetase. Biochem. Biophys. Res. Commun. 2006, 344, 1008-1016. [CrossRef] [PubMed]

22. Galluccio, M.; Brizio, C.; Torchetti, E.M.; Ferranti, P.; Gianazza, E.; Indiveri, C.; Barile, M. Over-expression in Escherichia coli, purification and characterization of isoform 2 of human FAD synthetase. Protein Expr. Purif. 2007, 52, 175-181. [CrossRef] [PubMed]

23. Serrano, A.; Sebastian, M.; Arilla-Luna, S.; Baquedano, S.; Herguedas, B.; Velazquez-Campoy, A.; Martinez-Julvez, M.; Medina, M. The trimer interface in the quaternary structure of the bifunctional prokaryotic FAD synthetase from Corynebacterium ammoniagenes. Sci. Rep. 2017, 7, 404. [CrossRef] [PubMed]

24. Serrano, A.; Sebastian, M.; Arilla-Luna, S.; Baquedano, S.; Pallares, M.C.; Lostao, A.; Herguedas, B.; Velazquez-Campoy, A.; Martinez-Julvez, M.; Medina, M. Quaternary organization in a bifunctional prokaryotic FAD synthetase: Involvement of an arginine at its adenylyltransferase module on the riboflavin kinase activity. Biochim. Biophys. Acta 2015, 1854, 897-906. [CrossRef] [PubMed]

25. Torchetti, E.M.; Bonomi, F.; Galluccio, M.; Gianazza, E.; Giancaspero, T.A.; Iametti, S.; Indiveri, C.; Barile, M. Human FAD synthase (isoform 2): A component of the machinery that delivers FAD to apo-flavoproteins. FEBS J. 2011, 278, 4434-4449. [CrossRef] [PubMed]

26. Miccolis, A.; Galluccio, M.; Nitride, C.; Giancaspero, T.A.; Ferranti, P.; Iametti, S.; Indiveri, C.; Bonomi, F.; Barile, M. Significance of redox-active cysteines in human FAD synthase isoform 2. Biochim. Biophys. Acta 2014, 1844, 2086-2095. [CrossRef] [PubMed]

27. Torchetti, E.M.; Brizio, C.; Colella, M.; Galluccio, M.; Giancaspero, T.A.; Indiveri, C.; Roberti, M.; Barile, M. Mitochondrial localization of human FAD synthetase isoform 1. Mitochondrion 2010, 10, 263-273. [CrossRef] [PubMed]

28. Giancaspero, T.A.; Galluccio, M.; Miccolis, A.; Leone, P.; Eberini, I.; Iametti, S.; Indiveri, C.; Barile, M. Human FAD synthase is a bi-functional enzyme with a FAD hydrolase activity in the molybdopterin binding domain. Biochem. Biophys. Res. Commun. 2015, 465, 443-449. [CrossRef] [PubMed]

29. Miccolis, A.; Galluccio, M.; Giancaspero, T.A.; Indiveri, C.; Barile, M. Bacterial over-expression and purification of the 3'phosphoadenosine 5' phosphosulfate (PAPS) reductase domain of human FAD synthase: Functional characterization and homology modeling. Int. J. Mol. Sci. 2012, 13, 16880-16898. [CrossRef] [PubMed] 
30. Olsen, R.K.; Konarikova, E.; Giancaspero, T.A.; Mosegaard, S.; Boczonadi, V.; Matakovic, L.; Veauville-Merllie, A.; Terrile, C.; Schwarzmayr, T.; Haack, T.B.; et al. Riboflavin-Responsive and -Non-responsive Mutations in FAD Synthase Cause Multiple Acyl-CoA Dehydrogenase and Combined Respiratory-Chain Deficiency. Am. J. Hum. Genet. 2016, 98, 1130-1145. [CrossRef] [PubMed]

31. Barile, M.; Giancaspero, T.A.; Leone, P.; Galluccio, M.; Indiveri, C. Riboflavin transport and metabolism in humans. J. Inherit. Metab. Dis. 2016, 39, 545-557. [CrossRef] [PubMed]

32. Olsen, R.K.; Olpin, S.E.; Andresen, B.S.; Miedzybrodzka, Z.H.; Pourfarzam, M.; Merinero, B.; Frerman, F.E.; Beresford, M.W.; Dean, J.C.; Cornelius, N.; et al. ETFDH mutations as a major cause of riboflavin-responsive multiple acyl-CoA dehydrogenation deficiency. Brain 2007, 130, 2045-2054. [CrossRef] [PubMed]

33. Rhead, W.; Roettger, V.; Marshall, T.; Amendt, B. Multiple acyl-coenzyme A dehydrogenation disorder responsive to riboflavin: Substrate oxidation, flavin metabolism, and flavoenzyme activities in fibroblasts. Pediatr. Res. 1993, 33, 129-135. [CrossRef] [PubMed]

34. Huerta, C.; Borek, D.; Machius, M.; Grishin, N.V.; Zhang, H. Structure and mechanism of a eukaryotic FMN adenylyltransferase. J. Mol. Biol. 2009, 389, 388-400. [CrossRef] [PubMed]

35. Giancaspero, T.A.; Locato, V.; Barile, M. A regulatory role of NAD redox status on flavin cofactor homeostasis in S. cerevisiae mitochondria. Oxid. Med. Cell. Longev. 2013, 2013, 612784. [CrossRef] [PubMed]

36. Bradford, M.M. A rapid and sensitive method for the quantitation of microgram quantities of protein utilizing the principle of protein-dye binding. Anal. Biochem. 1976, 72, 248-254. [CrossRef]

37. Barile, M.; Brizio, C.; Valenti, D.; De Virgilio, C.; Passarella, S. The riboflavin/FAD cycle in rat liver mitochondria. Eur. J. Biochem. 2000, 267, 4888-4900. [CrossRef] [PubMed]

38. Kelly, S.M.; Jess, T.J.; Price, N.C. How to study proteins by circular dichroism. Biochim. Biophys. Acta 2005, 1751, 119-139. [CrossRef] [PubMed]

39. Chen, Y.H.; Yang, J.T. A new approach to the calculation of secondary structures of globular proteins by optical rotatory dispersion and circular dichroism. Biochem. Biophys. Res. Commun. 1971, 44, 1285-1291. [CrossRef]

40. Barile, M.; Brizio, C.; De Virgilio, C.; Delfine, S.; Quagliariello, E.; Passarella, S. Flavin adenine dinucleotide and flavin mononucleotide metabolism in rat liver-The occurrence of FAD pyrophosphatase and FMN phosphohydrolase in isolated mitochondria. Eur. J. Biochem. 1997, 249, 777-785. [CrossRef] [PubMed]

41. Laemmli, U.K. Cleavage of structural proteins during the assembly of the head of bacteriophage T4. Nature 1970, 227, 680-685. [CrossRef] [PubMed]

42. Brizio, C.; Brandsch, R.; Bufano, D.; Pochini, L.; Indiveri, C.; Barile, M. Over-expression in Escherichia coli, functional characterization and refolding of rat dimethylglycine dehydrogenase. Protein Expr. Purif. 2004, 37, 434-442. [CrossRef] [PubMed]

Sample Availability: Not available.

(C) 2018 by the authors. Licensee MDPI, Basel, Switzerland. This article is an open access article distributed under the terms and conditions of the Creative Commons Attribution (CC BY) license (http:/ / creativecommons.org/licenses/by/4.0/). 